\title{
Quantitative susceptibility map from the patients with chronic heart failure
}

\author{
Jiun-Jie Wang ${ }^{1 *}$, Gigin Lin², Chao-Hung Wang ${ }^{3}$, Yu-Hsiang Juan², Pei-Ching Huang ${ }^{2}$ \\ From 19th Annual SCMR Scientific Sessions \\ Los Angeles, CA, USA. 27-30 January 2016
}

\begin{abstract}
Background
Iron deficiency can be related to patients with chronic heart failure. Although T2* mapping by MRI could be used as a non-invasive interrogator for the assessment of the iron loading, unfortunately the result is only with limited success. Quantitative susceptibility mapping is a further development using susceptibility weighted image. Previous studies suggested a strong linear co-relation between the iron concentration and the magnetic susceptibility. Therefore we used this technique to determine the iron loading and the measured susceptibility from the heart of patients with chronic heart failure.
\end{abstract}

\section{Methods}

50 patients with chronic heart failure were recruited from the local community. The study was approved by the institutional review board and is complied with the declaration of Helsinki. Informed consent was acquired from all participants. MR images were acquired using a $3 \mathrm{~T}$ scanner (Skyra, Siemensm Erlangen Germany). 3D fully flow compensated $\mathrm{T}^{*}$ weighted images were acquired with multiple TE of $2.21 ; 4.18 ; 6.15 ; 8.12 ; 10.09 ; 12.06 ; 14.3$ and 16 msec. Additional imaging parameters included TR(844.8 msec), Field of View (332 mm by $379 \mathrm{~mm}$ ) and matrix size (118 by 224). The quantitative susceptibility map was calculated by using software downloaded from Cornell MRI research Lab. Both the susceptibility weighted images and the $\mathrm{T} 2$ * map were calculated for comparison.

\section{Results}

Patients were divided into 2 groups by the left ventricle ejection fraction. The result showed that patients with the left ventricle ejection fraction smaller than $50 \%$, both the $\mathrm{T}_{2}^{*}$ and the quantitative susceptibility map were significantly smaller, when compared to patients with a larger left ventricle ejection fraction. The increased iron loading can be related to the cardiac function of the patients. Because $\mathrm{T}^{*}$ could vary from scanner to scanner, it is expected that quantitative susceptibility map can be a potentially useful image based biomarker for the early prognosis of chronic heart failure.

\section{Conclusions}

The quantitative susceptibility mapping in the diseased heart is feasible. It provides quantitative estimation of the iron loading and therefore can be of great interest in the studies related to iron deficiency in chronic heart failure.

\section{Authors' details}

'Medical Imaging and Radiological Sciences, ChangGung University, TaoYuan county, Taiwan. ${ }^{2}$ Medical Imaging and Intervention, ChangGung Memorial Hospital, TaoYuan county, Taiwan. ${ }^{3}$ ChangGung Memorial Hospital, Keelung, KeeLung, Taiwan.

Published: 27 January 2016

doi:10.1186/1532-429X-18-S1-P14

Cite this article as: Wang et al:: Quantitative susceptibility map from the patients with chronic heart failure. Journal of Cardiovascular Magnetic

Resonance 2016 18(Suppl 1):P14.

'Medical Imaging and Radiological Sciences, ChangGung University, TaoYuan county, Taiwan

Full list of author information is available at the end of the article 